\title{
Cirurgia plástica periodontal: gengivectomia e gengivoplastia: Relato de caso clínico
}

\author{
Periodontal plastic surgery: gingivectomy and gingivoplast: Clinical case report
}

Recebido: 20/05/2021 | Revisado: 25/05/2021 | Aceito: 28/05/2021 | Publicado: 29/05/2021

Letícia de Oliveira Domingues

Centro Universitário das Faculdades Metropolitanas Unidas, Brasil E-mail: ledomingues2@hotmail.com

Camille Lobato Marques

Centro Universitário das Faculdades Metropolitanas Unidas, Brasil

E-mail: camillelmarques@gmail.com

Caleb Shitsuka

Centro Universitário das Faculdades Metropolitanas Unidas, Brasil E-mail:cashitsuka@gmail.com

Renata Maria Mamprin Stopglia

Centro Universitário das Faculdades Metropolitanas Unidas, Brasil E-mail: liestudio@407.gmail.br

\begin{abstract}
Resumo
Uma aparência dentogengival agradável é bastante variável e depende da extensão da exposição da gengiva. A presença de um "sorriso gengival" aparente pode proporcionar grande insatisfação de ordem estética por parte do paciente. Na Periodontia, técnicas cirúrgicas podem ser empregadas como alternativas para este tipo de terapia, pois um sorriso agradável é parte importante na composição do aspecto geral do indivíduo. A gengivoplastia tem sido uma alternativa, para os casos de excesso do tecido gengival em que não há presença de doença periodontal. A gengivectomia é uma técnica cirúrgica de aplicabilidade limitada, que visa à eliminação do tecido gengival. Este trabalho tem como objetivo ilustrar, por meio de um caso clínico, as possibilidades de resolução estética utilizando a técnica da gengivectomiagengivoplastia.
\end{abstract}

Palavras-chave: Gengivectomia; Gengivoplastia; Sorriso Gengival.

\begin{abstract}
A pleasant dentogingival appearance is quite variable and depends on the extent of gum exposure. The presence of an apparent "gingival smile" can provide great aesthetic dissatisfaction on the part of the patient. In Periodontics, surgical techniques can be used as alternatives for this type of therapy, because a pleasant smile is an important part in the composition of the general aspect of the individual. Gingimoplasty has been an alternative to aesthetic therapies, for cases of excess gingival tissue in which there is no presence of periodontal disease. Often, the use of this technique requires the association of periodontal surgical techniques. Gingivectomy is a surgical technique of limited applicability, which aims at the elimination of gingival tissue (Grant et al., 1979). This study aims to illustrate, through a clinical case, the possibilities of aesthetic resolution using the gingivectomy-gingivectomy technique.
\end{abstract}

Keywords: Gingivectomy; Gingivoplasty; Gengival Smille.

\section{Introduçãa}

Ainda que a estética seja um conceito subjetivo que está diretamente relacionado aos padrões impostos pela sociedade e às variações individuais, um sorriso é considerado estético quando apresenta harmonia entre lábios, gengiva, forma, cor e disposição dos elementos dentários. Uma vez que o sorriso é uma formade expressão essencial para um indivíduo, o equilíbrio da relação dentogengival é fator de elevada importância na constituição de um sorriso estético, dentre eles se destacam alguns aspectos periodontais, como contorno, simetria, posicionamento gengival e extensão do tecido gengival exposto ${ }^{1,2}$.

Para um sorriso mais harmônico, é desejável uma exposição da margem gengival saudável e continua cerca de até 3 $\mathrm{mm}$ durante o sorriso natural, além de possuir extensa faixa de gengiva queratinizada, não obtendo essas características 
podemos considerar "sorriso gengival". Isso ocorre quando há excessiva exposição de gengiva durante o sorriso podendo ser causados por Erupção passiva alterada oucrescimento vertical excessivo da maxila ${ }^{3}$.

A etiologia do sorriso gengival é uma combinação de diversos fatores, desde alterações esqueléticas, dentárias, periodontais, labiais ou externas, estes fatores etiológicos podem surgir isoladamente ou em combinação ${ }^{4}$.

Sendo assim, pacientes com sorriso gengival, caracterizado por exposição excessiva da gengiva não possuem um sorriso considerado padrão, ocasionando grande insatisfação no mesmo, fazendo com que cada vez mais a busca por tratamentos e por um bom profissional aumente ${ }^{2}$.

Ao tratarmos de estética periodontal, existem algumas técnicas que permitem a harmonia do tecido gengival em relação a cor, forma e à sua arquitetura 5 .

Técnicas cirúrgicas são alternativas para este tipo de terapia, pois um sorriso agradável é parte importante na composição do aspecto geral do indivíduo ${ }^{6}$. Entre as diversas opções de tratamento incluem técnicas cujo fim é diminuir a quantidade de gengiva através da remodelação gengival e/ou óssea; diminuição da altura óssea

maxilar; reposicionamento dentário ortodôntico, ou a redução da capacidade de elevação ou função do lábio superior ${ }^{6}$. Técnicas cirúrgicas como a Gengivectomia e gengivoplastia podem ser empregadas como alternativas em busca de um sorriso agradável.

Somente após um exame clinico adequado e o correto diagnóstico acerca de qual fator etiológico está presente em cada caso, é que um adequado plano de tratamento pode ser proposto.

Este trabalho tem como propósito realizar uma revisão de literatura cientifica e,relatar um caso clinico de um paciente que apresenta sorriso gengival por erupção passiva alterada, abordando a periodontia a partir das técnicas cirúrgicas Gengivectomia e Gengivoplastia possibilitando um sorriso mais estético e harmônico.

\section{Metodologia}

Para a elaboração deste trabalho foi realizado buscas de artigoscientíficos em diversas plataformas de pesquisa científicas como PubMedUtilizou-se as palavras-chave: sorriso gengival, gengivoplastia e gengivectomia.

Através das palavras foi possível levantar e identificar os principais pontossobre o tema em questão.

\section{Revisão de Literatura}

\section{Sorriso Gengival}

A busca por um belo sorriso tem sido muito procurada dentro dos consultórios odontológicos por parte dos pacientes que, desejam resultados estéticos favoráveis, além da saúde bucal. No entanto para se alcançar um sorriso harmonioso é necessário haver simetria, entre as estruturas faciais, labiais, contorno gengival e dentes ${ }^{7}$.

Uma das características que têm despertado interesse na estética do sorriso éa quantidade de exposição gengival ao sorrir $^{8}$.

Certa quantidade à mostra de gengiva é aceitável para a estética, sendo considerada como um fator de jovialidade. Porém, ao expor mais de $3 \mathrm{~mm}$ de gengiva,o sorriso torna-se antiestético. Contemporaneamente a nomenclatura utilizada para essa situação clinica é "Sorriso gengival"'.

O sorriso gengival é causado por uma combinação de variáveis, tais como excesso vertical de maxila, maior habilidade muscular para elevar o lábio superior ao sorrir, espaço interlabial aumentado no repouso, sobremordida e sobressaliência aumentadas. 
Variáveis como o comprimento do lábio superior, ângulos dos planos mandibular e palatal parecem não influenciar no sorriso gengival ${ }^{10}$, porém, lábio superior curto e coroa clínica curta poderiam contribuir para a exposição gengival ${ }^{4}$

Uma avaliação adequada da zona estática requer uma examinação dos tecidosmoles e esqueléticos da face, seguida por uma avaliação intra-oral do tecido periodontal, dos dentes e sua relação com os lábios ${ }^{11}$. A posição natural da cabeça, que permite que o paciente esteja sentado ou de pé em frente ao clínico, é a posiçãoóptima para avaliar o plano frontal ${ }^{12}$. O clínico deve observar e examinar o sorriso dopaciente a uma distância considerada normal de conversação entre sujeitos.

A linha do sorriso deve também ser avaliada, é determinada pela posição dos lábios durante um sorriso natural e não forçado $^{13}$. Na literatura científica está proposto um método de avaliação da linha do sorriso e da exposição dentária em que o cirurgiãodentista tem à sua disposição, uma escala que lhe permite, visualmente durante o exame clínico, avaliar estes parâmetros. Este método tem critérios objetivos que permite uma avaliação formal e estandardizada, sendo de fácil e rápida aplicação. Nesta escala o sorriso pode ser classificado como alto, médio ou baixo, embora esta classificação tenha ligeiras variações de acordo com o estudo em causa e os seus autores. Segundo Van der Geld et al. (2011) uma linha do sorriso alta revela a totalidade da coroa clínica e uma banda contínua de gengiva com um mínimo de $1 \mathrm{~mm}$, um traço predominante no sexo feminino. Numa linha de sorriso média, $75 \%$ a $100 \%$ da coroa clínica é exposta assim como a gengiva interproximal. Uma linha de sorriso baixa expõe menos de $75 \%$ da coroa clínica, sendo esta última predominante no sexomasculino ${ }^{14}$. A grande maioria da população, $69 \%$, apresenta uma linha do sorriso média enquanto que uma linha do sorriso alta pode ser observada em $11 \%$ dos pacientes ${ }^{15}$.

O termo sorriso gengival é utilizado quando existe uma sobre exposição da gengiva maxilar durante o sorriso. Em casos severos essa sobre exposição pode servisível numa posição de repouso ${ }^{16}$. Tal como a escala que classifica a linha do sorrisovaria entre diferentes autores, também a distinção destes dois conceitos é distinta emdiferentes estudos. Liébart et al. (2004) afirmam que o sorriso gengival está presente quando temos uma exposição gengival superior a $2 \mathrm{~mm}^{17}$, Mazzuco et al. (2010) consideram uma exposição superior a $3 \mathrm{~mm}^{18}$ e Van der Geld et al. (2011) superior a $4 \mathrm{~mm}^{14}$.

Uma das indicações para o tratamento do sorriso gengival é quando o paciente apresenta erupção passiva alterada, nestes casos o paciente possui um crescimento da maxila e posicionamento dos lábios normais, no entanto expõe gengiva e coroas dentarias curtas ${ }^{19}$.

Nesse caso quando o tecido gengival cobre parcialmente a coroa dos dentes, o tratamento indicado é a cirurgia periodontal ressectiva Gengivectomia e Gengivoplastia ${ }^{7}$.

Na Odontologia as cirurgias plásticas periodontais estão disponíveis para uso corrente, restituindo a forma e função do tecido gengival alterado. Uma correta indicação dessas cirurgias levara ao sucesso clinico, com potencialidade de melhora estética e funcionalidade ${ }^{7}$.

É importante também que o profissional entenda que o principal objetivo do tratamento é satisfazer a exigência do paciente, considerando que a estética e a

função mastigatória variam de maneira considerável de um indivíduo para outro ${ }^{20}$. Para isso, atender as crescentes expectativas dos pacientes quanto a estética bucal devemos estar preparados para proporcionar, na medida do possível, os resultados estéticos desejáveis ${ }^{7}$.

\section{Gengivectomia}

A gengivectomia foi definida, em 1979, como uma excisão de tecido mole de uma bolsa periodontal patológica ${ }^{3}$. Widman (1981) publicou a técnica que foi chamadade "Retalho Original de Widman" onde descreveu a técnica de retalho para eliminação da bolsa periodontal com ou sem supuração e recontorno ósseo para estabelecer uma nova fisiologia para o osso alveolar $^{21}$. Neuman (1920) posteriormente propôs alterações à técnica original introduzindo incisão intrasulcular e acesso para 
melhor debridamento radicular ${ }^{23}$. Novamente Neuman (1982) publicou artigo demonstrando remoção do colarinho gengival após retalho mucoperiosteal seguido de nivelamento ósseo com broca esférica ${ }^{24}$.

A gengivectomia é realizada quando existe quantidade de tecido gengival suficiente para que, após a sua remoção, a margem gengival livre fique a $3 \mathrm{~mm}$ da crista óssea alveolar ${ }^{25}$. Segundo Abou-Arraj e colaboradores (2013), para a realizaçãode gengivectomia deverão estar presentes três principais condições ${ }^{26}$ : (1) localizaçãoda crista alveolar óssea em relação à JAC de, aproximadamente, 1,5 a $2 \mathrm{~mm}$, (2) espessura adequada de osso alveolar e (3) banda de gengiva queratinizada larga.

A distância entre a crista óssea e a junção amelocementaria que varia entre $1,5 \mathrm{~mm}$ a $2 \mathrm{~mm}$ é determinante na indicação de remodelação óssea, onde não houver esta distância deve ser feito a osteotomia para que haja espaço suficiente para a acomodação da inserção conjuntiva, epitélio juncional e sulco gengival (espaço biológico). Caso não se verifiquem estas condições, é provável que ocorram recidivas ${ }^{19}$.

A gengivectomia está relacionada com a remoção de gengiva inserida, marginal e papilar, na ausência de doenças periodontais. Também é indicada para eliminação de bolsas supra-ósseas, aumento de coroa clínica, eliminação de margens espessas e remoção de hiperplasias causadas por processos inflamatórios, hormonais, congênitos ou estimulado por medicamentos 25 .

Esta técnica não necessita a elevação do retalho cirúrgico (flapless) pois é uma técnica minimamente invasiva que permite a preservação do periósteo e fornece maiorsuprimento sanguíneo ao tecido, reduzindo a reabsorção óssea e contribuindo para acicatrização de tecidos, proporcionando um pós-operatório mais rápido e confortável para o paciente ${ }^{27}$.

\section{Gengivoplastia}

A gengivoplastia é uma cirurgia que corrige ou elimina deformidades gengivais, traumáticas ou de desenvolvimento, sendo considerada como o procedimento cirúrgico que proporciona o contorno gengival em espessura. É indicada em casos de ausência de doença periodontal e para correção estética, visando criar um contorno gengival harmônico, sulcos interdentais e remodelamentodas papilas interdentais ${ }^{28}$.

\section{Relato de Caso Clinico}

Paciente G.M, gênero masculino, 26 anos de idade procurou a Clínica Odontológica do Centro Universitário da Faculdades Metropolitanas Unidas -FMU- com o objetivo de melhorar a estética do seu sorriso. Durante a anamnese o paciente queixou-se da "insatisfação com relação ao tamanho de seus dentes e aspecto gengival" comprometendo assim a sua estética.

Após ter assinado o Termo de Consentimento Livre e Esclarecido (TCLE) o paciente foi submetido ao exame clínico onde pudemos observar, presença de dentescurtos, assimétricos, e excesso de tecido gengival nos dentes anteriores da maxila, compreendido entre os dentes 15 ao 25 (Figura1). 
Figura 1- Foto inicial.

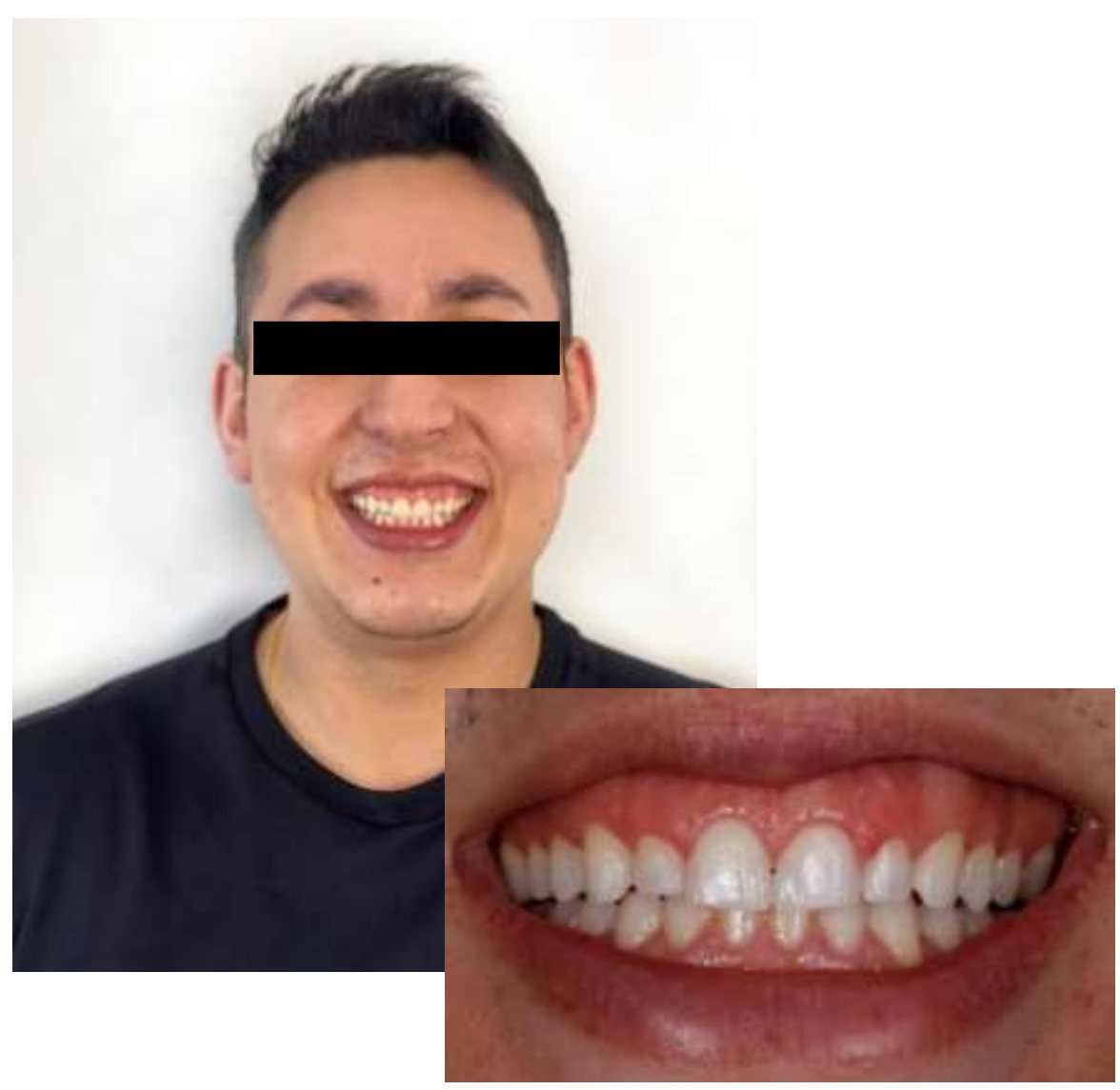

Fonte: Autores.

Ao realizamos os exames periodontais através da sondagem clínica foi possível identificar tecidos periodontais de suportes saudáveis, com ausência de sangramentogengival e ausência de placa visível (Figura 2).

Figura 2 - Tecidos periodontais de suportes saudáveis, com ausência de sangramentogengival e ausência de placa visível

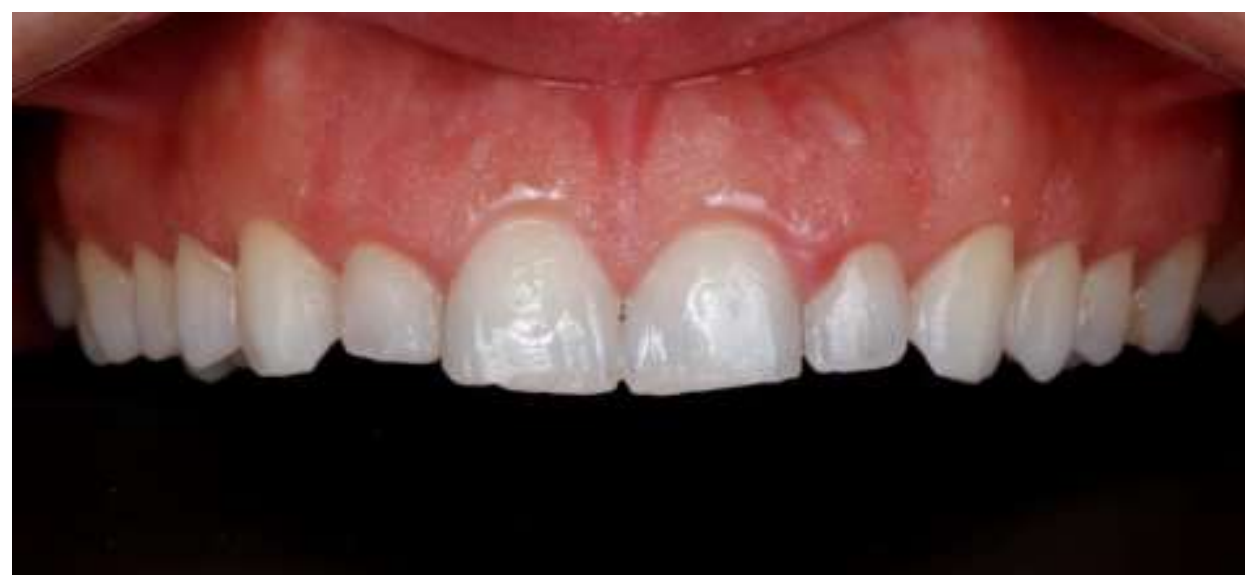

Fonte: Autores.

Para melhor precisão diagnóstica realizou-se o exame radiográfico e tomografiada maxila onde revelou não haver perdas ósseas ou qualquer alteração no periodontode sustentação, confirmando a etiologia de Erupção passiva alterada. (Figuras 3 e 4). 
e-Acadêmica, v. 2, n. 2, e012224, 2021

(CC BY 4.0) | ISSN 2675-8539 | DOI: http://dx.doi.org/10.52076/eacad-v2i2.24

Figura 3 - Radiografias, 14 periapicais.

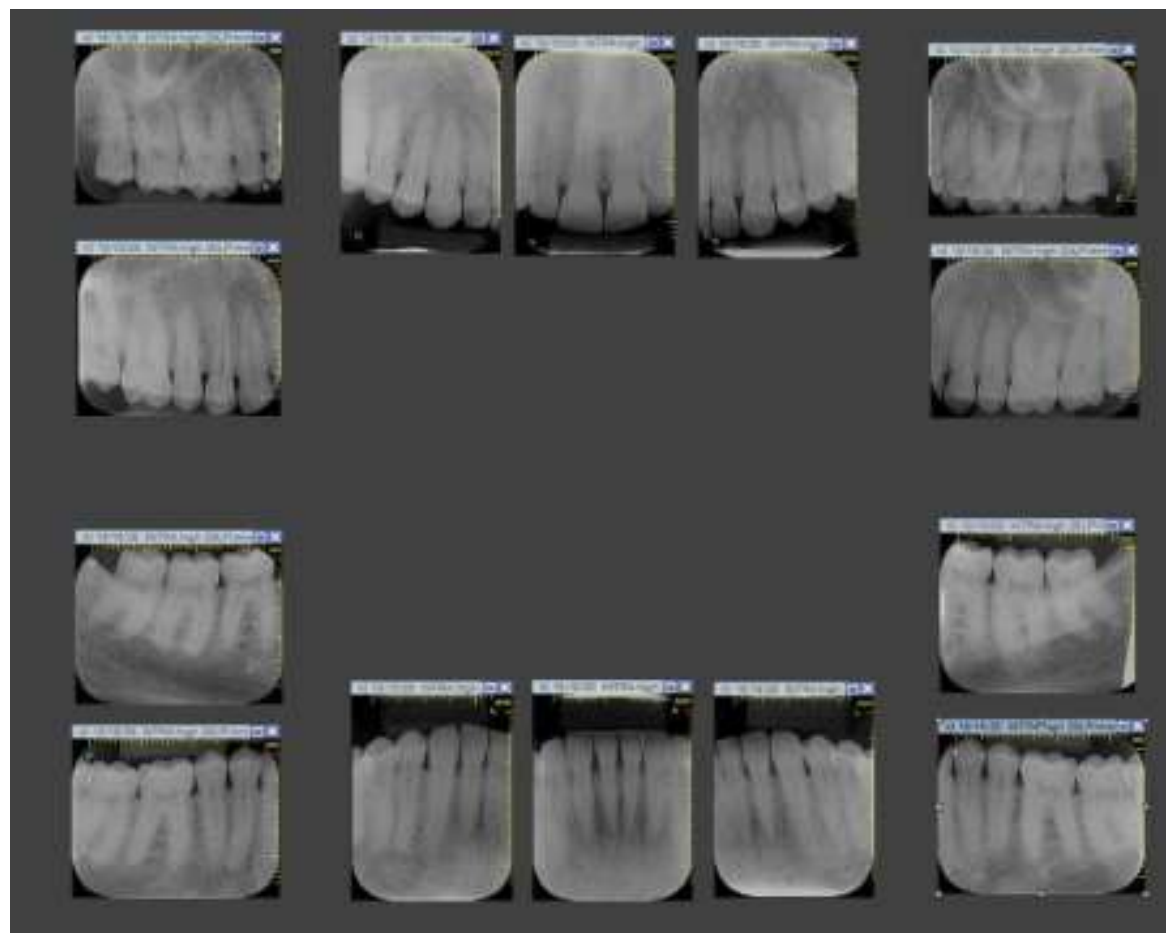

Fonte: Autores.

Figura 4 - Tomografia da maxila.

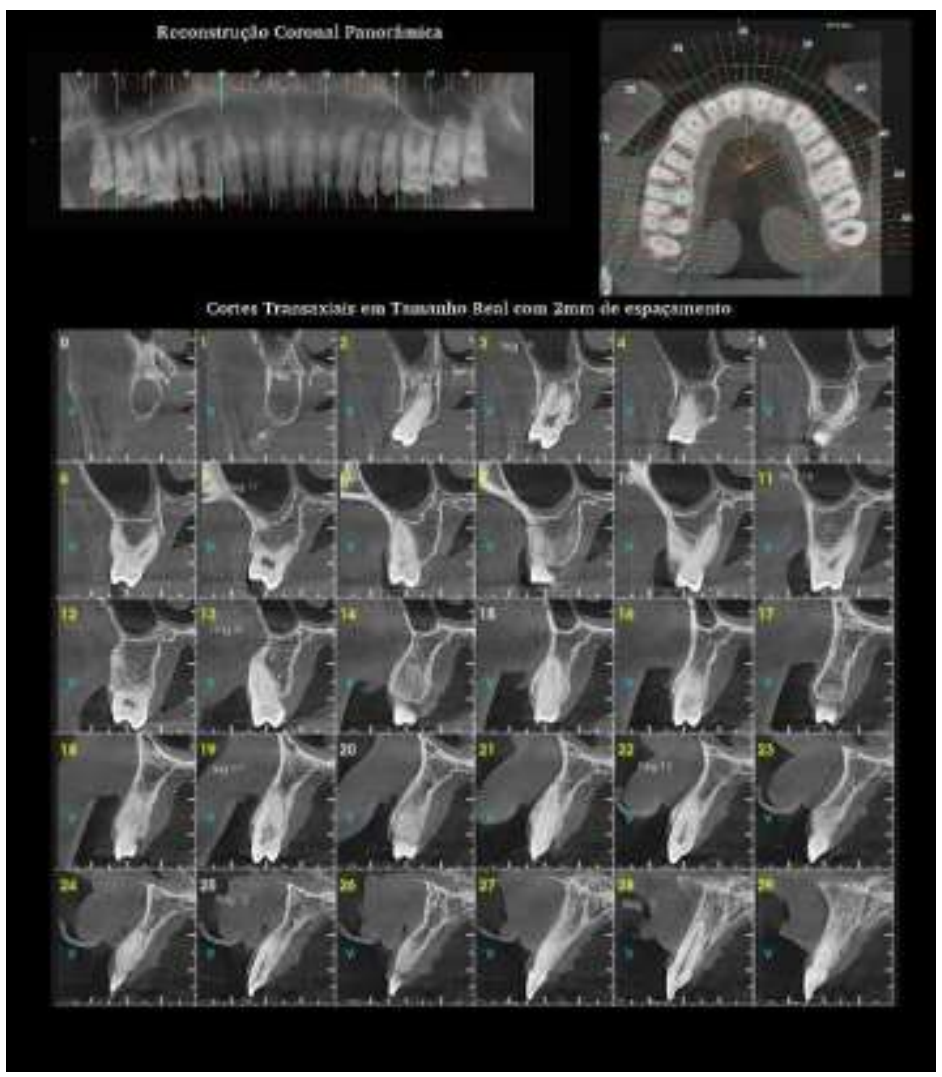

Fonte: Autores. 
Como foi observado relação desarmônica entre comprimento do dente e o rosto do paciente foi indicado a cirurgia de Gengivectomia/ Gengivoplastia de bisel interno para a correção e harmonia do sorriso, visto que o paciente apresenta saúde periodontal, profundidade de sondagem e nível de inserção adequados (Figura 5).

Figura 5 - Observação da relação desarmônica entre comprimento do dente e o rosto do paciente.

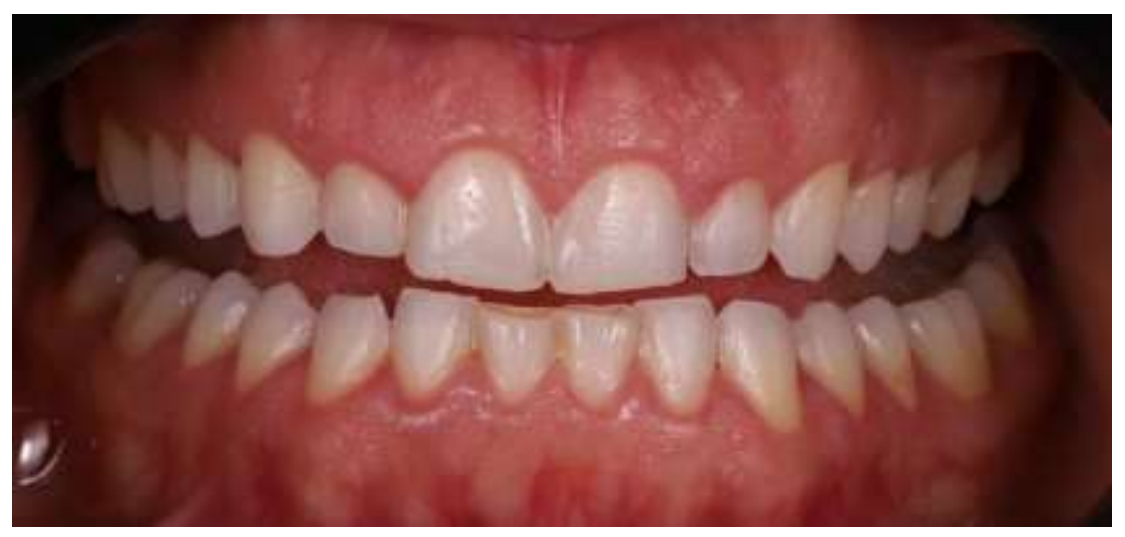

Fonte: Autores.

O paciente foi moldado com silicone de adição, posteriormente foi confeccionado um modelo de estudo com gesso especial que através do enceramentodiagnóstico projetou-se o aumento de coroa clínico com todas as particularidades estéticas e funcionais. As medidas obtidas através da sondagem transgengival foram transferidas para o modelo com lápis cópia, obedecendo os princípios estéticos dos Zênites. (Figura 6)

Foi confeccionado sobre o modelo uma placa de acrílico/acetato para servir como guia cirúrgico, a borda do guia foi recortado com o objetivo de delimitar onde seencontra o osso. (Faria et al. 2015) (Figura 7)

Figura 6 - Modelo com lápis cópia.

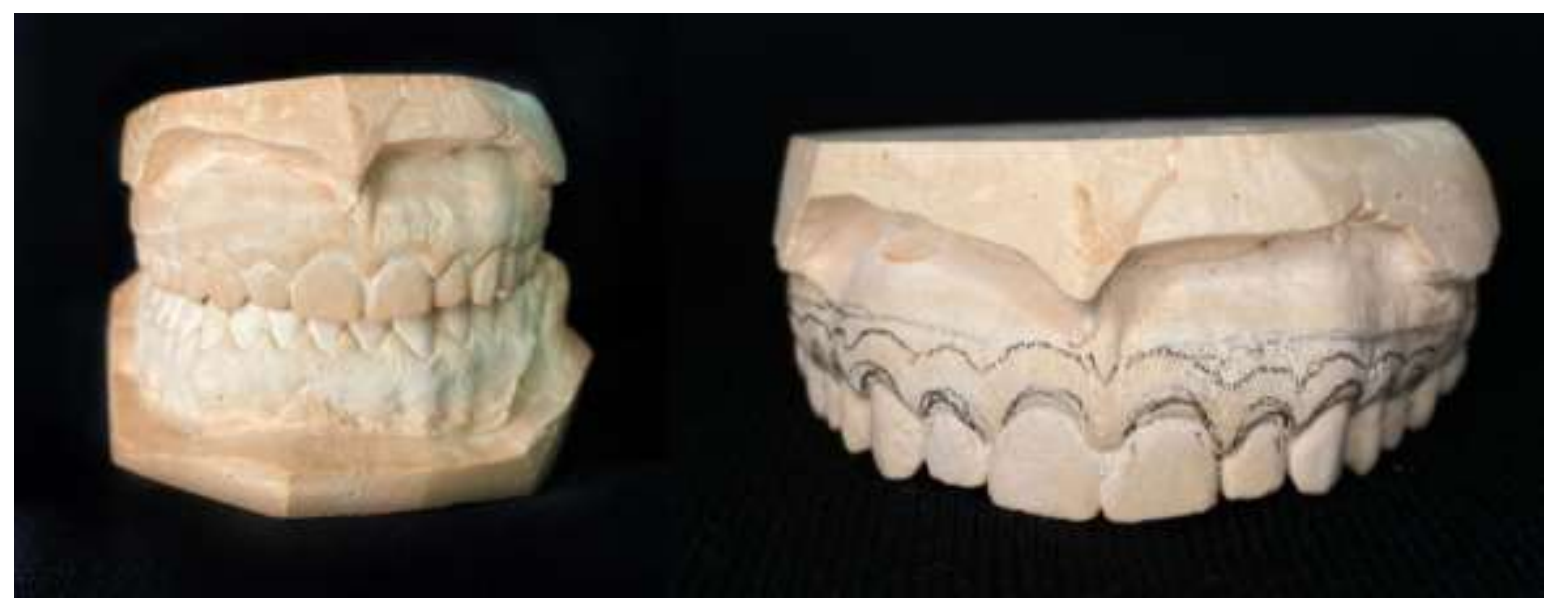

Fonte: Autores. 
Figura 7 - Modelo com placa de acrílico.

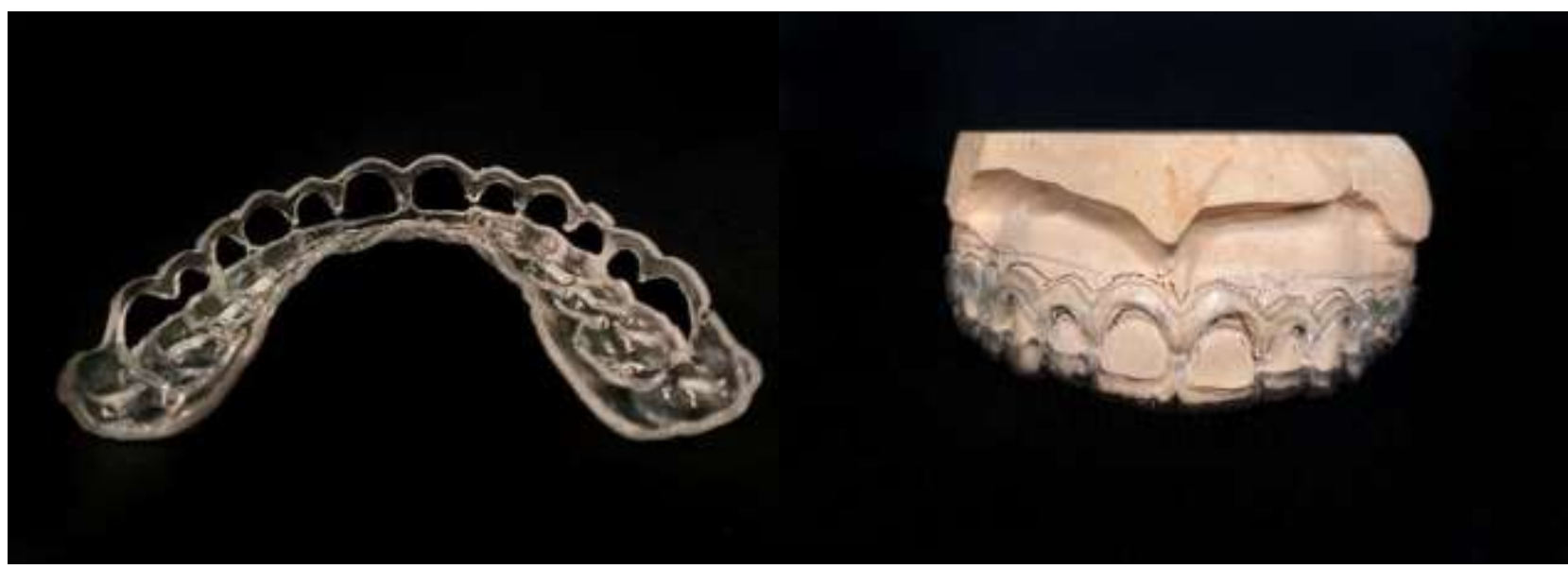

Fonte: Autores.

Técnicas cirurgicas

Previamente a cirurgia o paciente foi orientado a realizar a assepsia com bochecho de solução de digluconato de clorexidina $0,12 \%$ visando diminuir a bacteremia na cavidade oral durante a cirurgia.

Iniciamos o procedimento cirúrgico realizando a anestesia local pela técnica infiltrativa com mepivacaina a 2\% com epinefrina 1:100.000. (DFL, apud Tentrin, 2018).

Foi colocado o guia cirúrgico para guiar o trajeto da incisão primaria, que seguiu o delineamento com uma lâmina oftálmica (mani) dando contorno biselado nas margens gengivais criando um colar de tecido mole (Figuras 8 e 9).

Figura 8 - Guia cirúrgico.

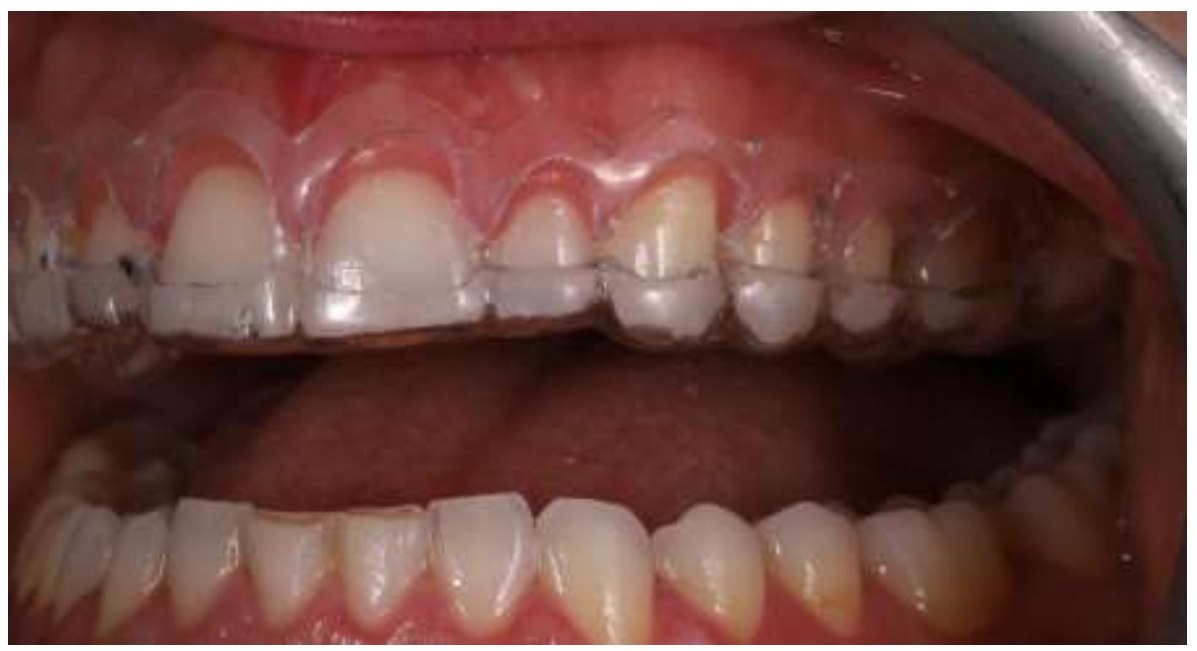

Fonte: Autores. 
Figura 9 - Guia cirúrgico.

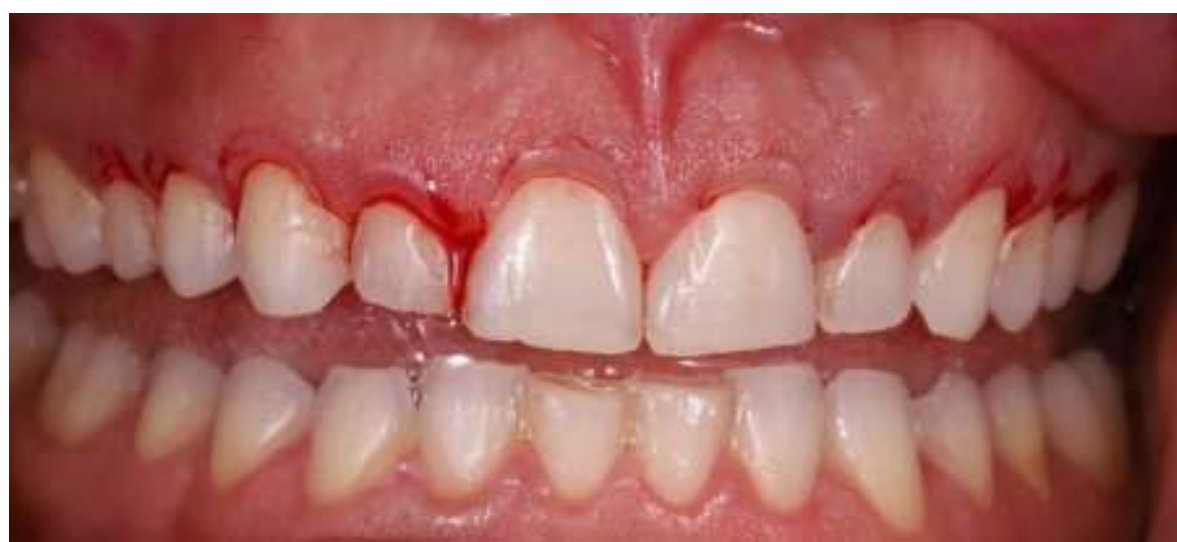

Fonte: Autores.

Em seguida retirou-se o guia cirúrgico e com a mesma lâmina oftálmica realizou-se a incisão secundaria a fim de remover o excesso (colar) de tecido gengival proporcionando remodelamento e alisamento estético.

Com o auxílio de uma lâmina 15 acoplada a um cabo de bisturi (Bard Parker) foi realizado o descolamento e reposicionamento do retalho.

Dessa forma, após a remoção do colarinho gengival foi realizada nova sondagem transgengival para a localização da distância da junção amelocementária e crista óssea dos dentes envolvidos confirmando aintegridade do espaço biológico.

Suturas do tipo colchoeiro vertical foram realizadas com fio de polipropileno 6-0 (monofilamentado) e agulha 3/8 circular com ponta triangular para estabilização do tecido gengival (Figura 10).

Figura 10 - Suturas tipo colchoeiro vertical.

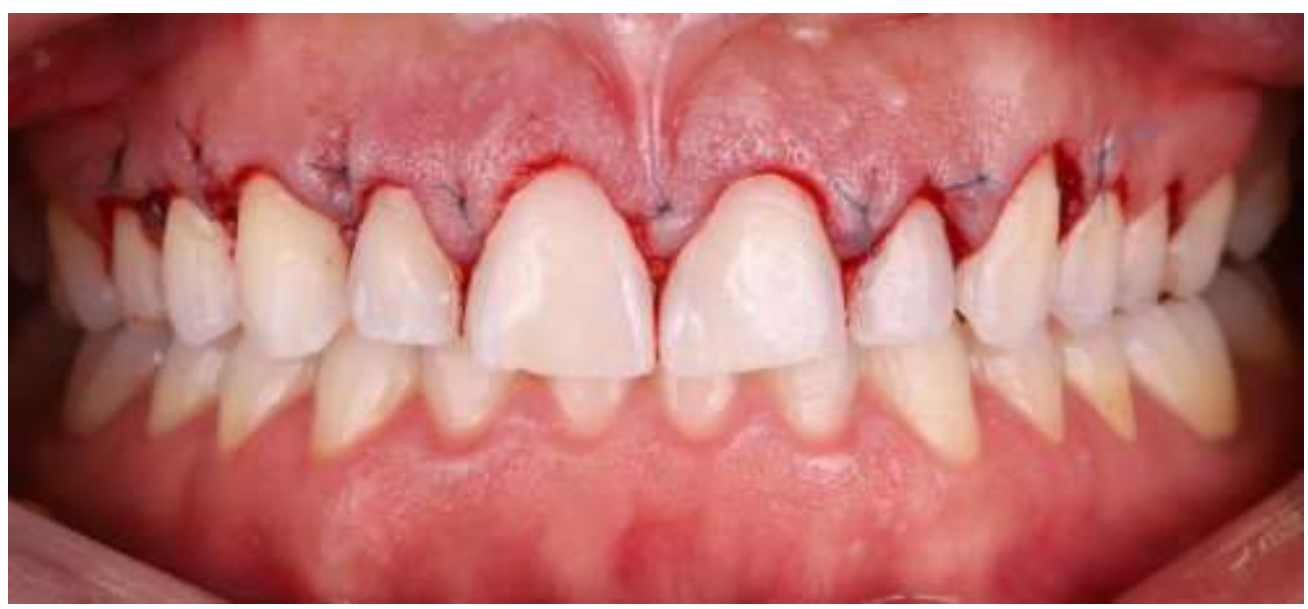

Fonte: Autores.

Seguiu-se irrigando com soro fisiológico e compressão ra a realização dahemostasia

Também foram realizadas orientações pós-operatório: após quarenta e oito horas fazer aplicações de digluconato de clorexidina $0,12 \%$ com cotonete umedecido três vezes ao dia no período de sete dias, escovação suave na região da cirurgia e optar por alimentos pastosos e frios.

Foi prescrita medicação analgésica (dipirona 50mg) embora no pós-operatório o paciente não tenha relatado nenhuma sintomatologia dolorosa. 
Após quinze dias removeu-se os pontos podendo notar-se bom quadro clinico e, após quatro meses o paciente retornou para acompanhamento, relatando grande satisfação, resultados que foram confirmados neste retorno, não havendo necessidade de reparos (Figura 11).

Figura 11 - Aspecto final do paciente.

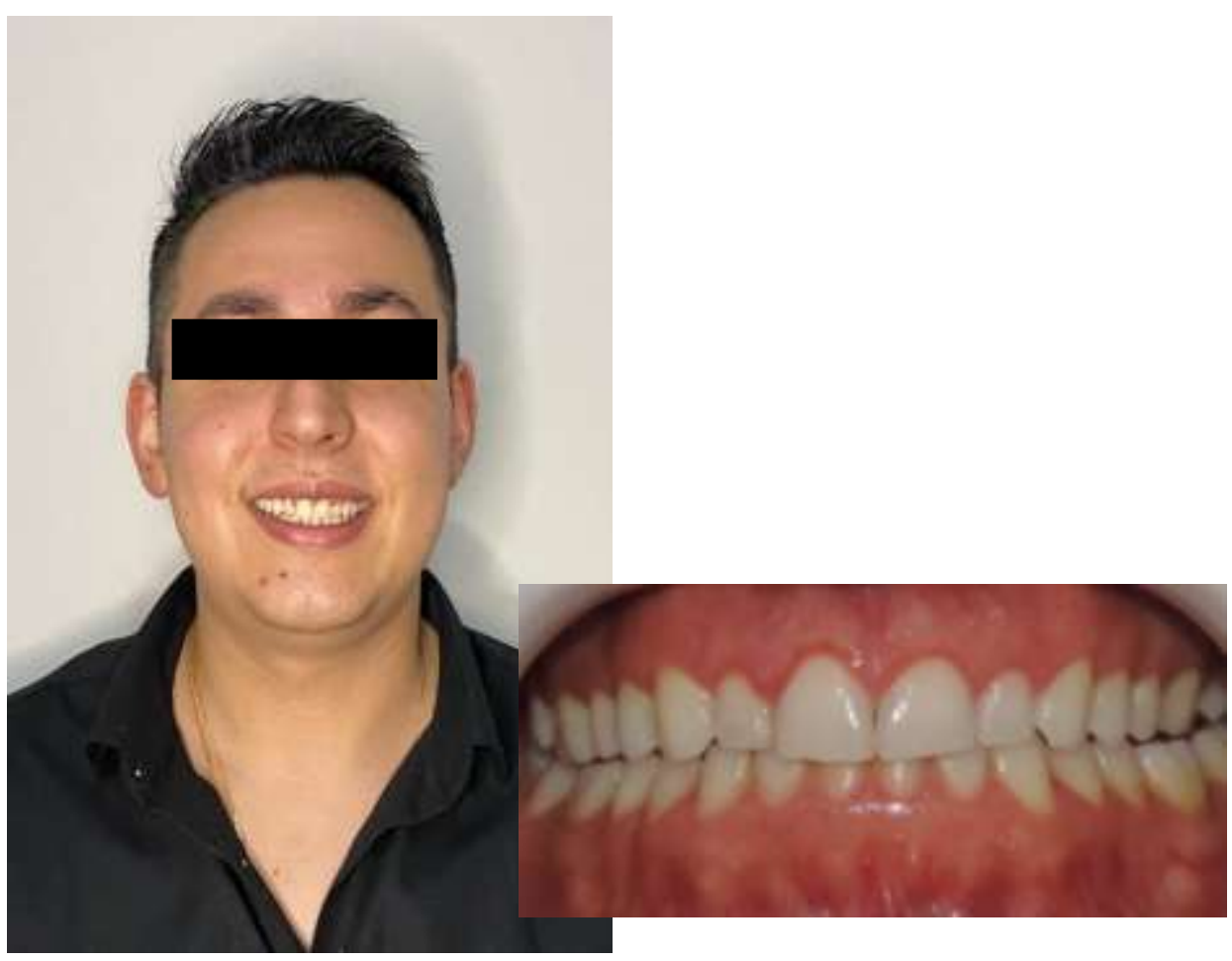

Fonte: Autores.

Com a finalização do caso clinico ficou nítida a melhora na estética e harmonia do sorriso, notando-se a importância da técnica ressectiva utilizada, promovendo a completa satisfação do paciente.

\section{Discussão}

Diversos autores apontam que os pacientes procuram um profissional embusca de um sorriso perfeito, isto é um sorriso que se adeque aos padrões estéticos: equilibrados e harmoniosos ${ }^{29}$. O padrão preestabelecido como osorriso ideal é aquele que possui tanto uma correlação harmoniosa entre a formae as cores dos dentes quanto uma boa proporção entre lábio e gengiva ${ }^{29}$. $\mathrm{Um}$

sorriso gengival afeta o estado estético e psicológico, pois geralmente diminui aautoconfiança, levando a esconder ou controlar o sorriso $^{30}$.

Com a odontologia contemporânea fica mais fácil obter uma estrutura dentária sadia, pois apresenta novos procedimentos que possibilitem a estéticadentofacial ${ }^{31}$.

Em paciente cujo o sorriso não lhe agrada esteticamente, seja por casos de excesso do tecido gengival sem a presença de doença periodontal a gengivoplastia tem sido uma alternativa às terapias estéticas. Este procedimentocirúrgico tem indicação para eliminação de bolsas supra ósseas ou falsas bolsas,crateras interproximais gengivais, aumento de coroa clínica, eliminação de margens espessas e remoção de crescimento gengival causado por processos inflamatórios, hormonais, congênitos ou induzidos por medicamentos ${ }^{32}$

A fim de que seja eleita a melhor técnica cirúrgica e/ou restauradora para casos específicos de sorriso gengival, é necessário que o profissional realize umcorreto diagnóstico e planejamento do caso, devolvendo a harmonia facial do paciente através do conjunto "lábios- gengiva-dentes"33. 
Para uma adequada reabilitação bucal pode ser alcançada considerando-se vários fatores, dentre eles os princípios biológicos (respeito e contribuição à preservação dos tecidos gengivais saudáveis adjacentes, às restaurações e próteses dentárias); mecânicos (retenção e adesão) e estéticos (dependente devariáveis socioculturais) ${ }^{33}$.

No presente caso clínico o paciente relatou a insatisfação devido àestética dentária, pois tinha a presença de dentes curtos, assimétricos, e excesso de tecido gengival nos dentes anteriores da maxila, compreendido entre os dentes 15 ao 25. Realizado o diagnóstico, a cirurgia plástica periodontal foi uma opção terapêutica previsível, segura e importante na resolução dos problemas estéticos e funcionais dos pacientes ${ }^{34}$

Por isso fez-se uma gengivoplastia no paciente que é um procedimento cirúrgico de alteração do contorno gengival visando a diminuir a margem gengival, criando contorno gengival recortado, afinando a gengiva inserida, criando sulcos interdentais verticais e remodelando a papila interdentária para criar espaço para a passagem de alimentos ${ }^{35}$.

Antes, porém, fez-se um exame adequado do lábio na avaliação estéticado paciente periodontal, análise das estruturas dentofaciais e exame odontológico completo e adequado ${ }^{36}$. Também foi avaliado os aspectos de simetria facial, linha do sorriso alta e baixa, simetria dos lábios/face e exposiçãogengival quando sorrindo, altura e harmonia das margens gengivais, tamanho eproporção dos dentes, dentre outros, também devem ser registrados ${ }^{37}$.

O presente caso clínico atingiu um resultado clínico muito satisfatório no quesito de melhorias estéticas da relação dentogengival, uma vez que o pacientepossuía sorriso alto e amplo, lábios finos, desalinhamento dental e exposição gengival maior que $3 \mathrm{~mm}$ ao sorrir, caracterizada por erupção passiva alterada em função dos dentes curtos e assimétricos.

\section{Conclusão}

Através da revisão de literatura sobre o tema, pode-se concluir a importância da estética do sorriso na vida dos indivíduos atualmente, a importância de um correto exame clínico, o qual se avalia tecidos moles e esqueléticos da face, seguido por uma avaliação rígida intra oral do tecido periodontal, dos dentes, da sua relação com a gengiva e a linha do sorriso, alémde descobrir a etiologia, para que então, possa ser feito o melhor planejamento e a escolha adequada do tratamento para o caso.

Sendo assim, quando as técnicas são empregadas corretamente, têm-se grande satisfação do paciente. Portanto, torna imprescindível uma boa avaliaçãoe maestria na utilização dos métodos descritos anteriormente que gerem, não somente, um resultado funcional positivo, como também a melhoria estética.

Por meio do caso relatado, pode-se concluir que é fundamental para queo resultado cirúrgico ocorra com exatidão e impecabilidade, um bom exame clinico, planejamento e exames complementares como radiografia e tomografia. Assim como também se faz fundamental a utilização dos materiais adequados eda técnica empregada com precisão do passo a passo, além da indispensável colaboração do paciente com o pós-operatório.

\section{Referências}

1-Grant DA, Stern IB, Everett FG. Gengivectomy and gengivoplasty. In:Periodontics: a concepttheory and practice. $4^{\mathrm{a}}$ ed. St Louis: C.V. Mosby; 1979. Cap.28. p.446-72. In: Souza, CP, Garzon, ACM, Sampaio, JEC. EstéticaPeriodontal: Relato de um caso. Revista Brasileira de Cirurgia e Periodontia, 2003; 1(4):262-7. [acesso 14 set.2020] Disponível em:https://www.dtscience.com/wp- content/uploads/2015/10/Est\%C3\%A9tica- Periodontal-Relato-de-umCaso.pdf

2-Faria, G J et al. A importância do planejamento multidiscipilinar para correção do sorriso gengival: Relato de caso clínico.-DOI: http://dx. doi. org/10.15600/2238-1236/fol. v25n1p61-65. Revista da Faculdade de Odontologia de Lins, v. 25, n. 1, p. 61-66, 2015.In: Kitayama, Suemy Simplício. Diagnóstico de Tratamento do Sorriso Gengival. Universidade federal do Rio Grande do Norte. [acesso 10 nov 2020 ]. Disponível em < https://monografias.ufrn.br/jspui/bitstream/123456789/4814/1/\%5B2016.2\%5D\%20Diagn\%C3\%B3stico\%20e\%20tratamento\%20do\%20sorriso\%20gengival. PDF>

3-Trentin, M S et al. Correção de assimetria dentogengival com finalidade estética: Relato de caso clínico. SALUSVITA, Bauru, v.37, n. 3, p. 671-684, 2018. 
4-Bidra, AV, Agar JR, Parel SM. Management of patients with excessive gingivaldisplay for maxillary complete arch fixed implant-supported prostheses. J Prosthet Dent Nov 2012. 8: 324-31. In: Pinto, TB. Técnicas de Correção doSorriso Gengival. [acesso $16 \quad$ set.2020] Disponível em: https://repositorio.ul.pt/bitstream/10451/26239/1/ulfmd06056_tm_Tiffany_Pinto.pdf.

5-Monnet-corti, V.; Borghetti, A. Estética do periodonto. In: Borghetti A, Monnet-Corti V. Cirurgia plástica periodontal. Porto Alegre: Artmed, p.98-112, 2002. . In: Álvaro NLA, Oliveira CMG. Gengivectomia e Gengivoplastia: Em Busca ao "Sorriso Perfeito". [acesso 14 set.2020] Disponível em: https://www.uninovafapi.edu.br/arquivos_academicos/repositorio_Biblioteca/odo ntologia/20191/ASPECTOS\%20GENGIVAIS \%20PARA\%20OTIMIZA \% C3\% 87\%C3\%830\%20DO\%20SORRISO-\%20REVIS\%C3\%830\%20DE\%20LITERATURA.pdf

6-Sousa, C. P.; Garzon, A. C. D. M.; Sampaio, J. E. C. Estética periodontal: relato de um caso. Rev Bras Cir Period, v. 1, p. 262-267, 2003.bb. In: Álvaro NLA, Oliveira CMG. Gengivectomia e Gengivoplastia: Em Busca ao "Sorriso Perfeito".[acesso $14 \quad$ set.2020] https://www.uninovafapi.edu.br/arquivos_academicos/repositorio_Biblioteca/odontologia/20191 /ASPECTOS\%20GENGIVAIS\% 20PARA\% 20OTIM IZA\% C3\%87\%C3\%830\%20DO\%20SORRISO-\%20REVIS\%C3\%830\%20DE\%20LITERATURA.pdf

7-Oliveira, SAR de et al. Cirurgia Periodontal Ressectiva Valorizando o SorrisoGengival: Relato de Caso Clínico. Colloquium Vitae,jul/dez 2012 4(2): 118 128. DOI: 10.5747/cv.2012.v004.n2.v069c .Faculdade de Odontologia daUNOESTE,Presidente Prudente,SP.

8-Reddy MS. Achieving gingival esthetics. J Am Dent Assoc. 2003 Mar;134(3):295-304. In: Mendes, APM. Sorriso gengival: Etiologia, diagnóstico e opções de tratamento. [acesso 25 set.2020] Disponível:https://repositorio.ul.pt/ bitstream/10451/27219/1/ ulfmd07095_tm_Ana_Mendes. pdf.

9-Seixas, MR; Costa-Pinto, RA; Araújo, TM. Checklist dos aspectos estéticos a serem considerados no diagnóstico e tratamento do sorriso gengival. Dental Press J Orthod, v. 16, n. 2, p. 131-57, 2011. In: Kitayama, SS. Diagnóstico e tratamento do sorriso gengival. [acesso 16 set.2020] Disponível em: https://monografias.ufrn.br/jspui/bitstream/123456789/4814/1/\%5B2016.2\%5D \%20Diagn\%C3\%B3stico\%20e\%20tratamento\%20do\%20sorriso\%20gengival.P $\mathrm{DF}$

10-Ahmad I. Anterior dental aesthetics: Gingival perspective. Br Dent J. 2005d Aug 27; 199(4):195-202. In: Mendes, APM. Sorriso gengival: Etiologia,diagnóstico e opções de tratamento. [acesso 18 set.2020] Disponível: https://repositorio.ul.pt /bitstream /10451/27219/1 /ulfmd07095_ tm_Ana_Mendes. pdf.

11-Panossian AJ, Block MS. Evaluation of the smile. Facial and dental considerations. J Oral Maxillofac Surg 2010; 68:547-554. In: Mendes, APM. Sorriso gengival: Etiologia, diagnóstico e opções de tratamento. [acesso 20 set.2020]. Disponível: https://repositorio.ul.pt/bitstream /10451/27219/1/ ulfmd07095_tm_ Ana_Mendes.pdf

12-Hasanreisoglu U, Berksun S, Aras K, Arslan I. An analysis of maxillaryanterior teeth: facial and dental proportions. J Prosthet Dent. 2005 Dec; 94(6):530-8. In: Mendes, APM. Sorriso gengival: Etiologia, diagnóstico e opçõesde $\quad$ tratamento [acesso 20 set.2020]. Disponível: https://repositorio.ul.pt/bitstream/10451/27219/1/ulfmd07095_tm_Ana_Mendes.pdf

13-Reddy MS. Achieving gingival esthetics. J Am Dent Assoc. 2003 Mar; 134(3):295-304. In: Mendes, APM. Sorriso gengival: Etiologia diagnóstico eopções de tratamento. [acesso 25 set.2020] Disponível:https://repositorio.ul.pt/bitstream/ 10451/27219/1/ulfmd07095_tm _Ana_ Mendes. pdf.

14-Van der Geld P, Oosterveld P, Schols J, Kuijpers-Jagtman AM. Smile line assessment comparing quantitative measurement and visual estimation. Am J Orthod Dentofacial Orthop. 2011 Feb; 139(2):174-80. In: Mendes, APM. Sorrisogengival: Etiologia, diagnóstico e opções de tratamento. [acesso 30 set.2020]Disponível: https://repositorio.ul.pt/bitstream/10451/27219/1/ulfmd07095_tm_Ana_Mendes. pdf.

15-Ravon NA, Handelsman M, Levine D. Multudisciplinary care: periodontal aspects to treatment planning the anterior esthetic zone. J Calif Dent Assoc. 2008 Aug; 36(8):575-84. In: Mendes, APM. Sorriso gengival: Etiologia,diagnóstico Disponível:https://repositorio.ul.pt/bitstream/10451/27219/1/ulfmd07095_tm_Ana_Mendes. pdf. e opções de tratamento. [acesso $30 \quad$ set.2020]

16-Silberberg N, Goldstein M, Smidt A. Excessive gingival display - Etiology,diagnosis, and treatment modalities.Quintessence Int. 2009 Nov-Dec; 40(10):809-18. In: Mendes, APM. Sorriso gengival: Etiologia, diagnóstico eopções de tratamento. [acesso 30et.2020] Disponível: https://repositorio.ul.pt/bitstream/10451/27219/1/ulfmd07095_tm_Ana_Mendes. pdf.

17-Liébart MF, Fouque-Deruelle C, Santini A, Dillier FL, Monnet-Corti V, Glise JM, Borghetti A. Smile line and periodontium visibility. Periodont Pract today.2004; 1:17-25. In: Mendes, APM. Sorriso gengival: Etiologia, diagnóstico eopções de tratamento.[acesso 30 set.2020] Disponível: https://repositorio.ul.pt/bitstream/10451/27219/1/ulfmd07095_tm_Ana_Mendes. pdf.

18-Mazzuco R, Hexsel D. Gummy smile and botulinum toxin: a new approach based on the gingival exposure area. J Am Acad Dermatol. 2010 Dec; 63(6):1042-51. In: Mendes, APM. Sorriso gengival: Etiologia, diagnóstico eopções de tratamento. [acesso 30 set.2020] Disponível:

https://repositorio.ul.pt/bitstream/10451/27219/1/ulfmd07095_tm_Ana_Mendes. pdf.

19-Brilhante FV, Araújo RJG, Mattos JL, Damasceno JM, Frota LV, Pinto, RAPC. Cirurgia periodontal estética em dentes anteriores. [acesso 25 set.2020] Disponível em: http://pesquisa.bvsalud.org/portal/resource/pt/biblio-874866.

20-Barros-Silva, D et al. Cirurgia Plástica Periodontal para Otimização da Harmonia Dentogengival - Relato de Caso Clínico Periodontal plastic surgery to optimize the harmony dentogengival - case report.Universidade Camilo CasteloBranco - UNICASTELO, São Paulo, SP. Rev.Braz J Health, 2010; 1: 31 -36

21-Widman, L (1918). The operative treatment of pyorrhea alveolaris. A new surgical method. Svensk Tandläkaretidskrift (reviwed in British Dental Journal) 1, 293, 1920. In: Brilhante FV, Araújo RJG, Mattos JL, Damasceno JM, Frota LV, Pinto, RAPC. Cirurgia periodontal estética em dentes anteriores. [acesso 25 set.2020]Disponível em: http://pesquisa.bvsalud.org/portal/resource/pt/biblio- 874866.

22-Hasanreisoglu U, Berksun S, Aras K, Arslan I. An analysis of maxillary anteriorteeth: facial and dental proportions. J Prosthet Dent. 2005 Dec; 94(6):530-8. In: Mendes, APM. Sorriso gengival: Etiologia, diagnóstico e opções de tratamento. [acesso20set.2020]. Disponível: https://repositorio.ul.pt/bitstream/10451/27219/1/ulfmd07095_tm_Ana_Mendes.pdf 
23-Neuman (1920) D. Alveolar-pyorrhöe und ihre Behndlung,3rd edn. Berlin: Herman Meusser, Kirkland O(1931). The suppurative periodontal pus pocket; its treatment by the modified flap operation. Jounal of American Dental Association 18, 1462-1479.9. Neuman R. A pioneer in periodontal flap surgery. J Periodontol.1982 Jul;53(7):456-9. In: Brilhante FV, Araújo RJG, Mattos JL, Damasceno JM, Frota LV, Pinto, RAPC. Cirurgia periodontal estética em dentes anteriores. [acesso 25 set.2020] Disponível em:http://pesquisa.bvsalud.org/portal/resource/pt/biblio-874866.

24-Neuman R. A pioneer in periodontal flap surgery. J Periodontol.1982 Jul;53(7):456-9. In: Brilhante FV, Araújo RJG, Mattos JL, Damasceno JM, Frota LV, Pinto, RAPC.Cirurgia periodontal estética em dentes anteriores. [acesso 25 set.2020]Disponível em: http://pesquisa.bvsalud.org/portal/resource/pt/biblio874866.

25-Ribeiro, F V. et al. Open-flap versus flapless esthetic crown lengthening: 12- month clinical outcomes of a randomized controlled clinical trial. Journal of periodontology, v. 85, n. 4, p. 536-544, 2014. In: Kitayama, SS. Diagnóstico e tratamento do sorriso gengival. [acesso 16 set.2020] Disponível em: https://monografias.ufrn.br/jspui/bitstream/123456789/4814/1/\%5B2016.2\%5D\%20Diagn\%C3\%B3stico\%20e\%20tratamento\%20do\%20sorriso\%20gengival.P $\mathrm{DF}$

26-Abou - Arraj, R V.; Souccar, N M. Periodontal treatment of excessive gingival display. In: Seminars in Orthodontics. WB Saunders, 2013. p. 267-278. In: Kitayama, Suemy Simplício. Diagnóstico de Tratamento do Sorriso Gengival. Universidade federal do Rio Grande do Norte. [acesso 10 nov 2020]. Disponível

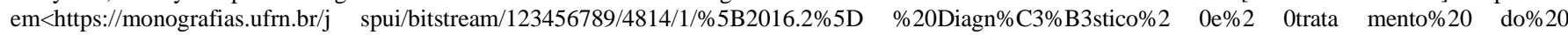
sorriso\%20gengival.P DF>

27-Pontoriero R, Lindhe J. Guided tissue regeneration in the treatment of degree II furcations in maxillary molars. J Clin Periodontol 1995;22:756-63.In: Desjardins, Marina Passarela. Utilização de enxerto ósseo autógeno para tratamento de lesão de bifurcação proximal Classe II Revista PerioNews, 20115(5):540-4

28-Tjan, A. H.; MIller, G. D.; The, J. G. Some esthetic factors in a smile. J Prost Dent., v. 51, p. 24-28, 1984. In: Álvaro NLA, Oliveira CMG. Gengivectomia e Gengivoplastia: Em Busca a "Sorriso Perfeito". [acesso 14 set.2020] Disponível em: https://www.uninovafapi.edu.br/arquivos_academicos/repositorio_Biblioteca/odo ntologia/20191/ASPECTOS\%20GENGIVAIS \%20PARA\%20OTIMIZA\% C3\%87\%C3\%830\%20DO\%20SORRISO-\%20REVIS\%C3\%830\%20DE\%20LITERATURA.pdf

29-Touati B. Defining form and position. Pract Periodonttics Aesthet Dent. 1998;10:800-7.In: Sousa, SJB et al. Cirurgia Plástica Periodontal para Correçãode Sorriso Gengival Associada à Restauração em Resina Composta: Relato de Caso Clínico. Ver. Odontol Bras Central 2010;19(51). Acesso [10 nov 2020] Disponível em: https://www.robrac.org.br/seer/index.php/ROBRAC/article/view/501

30-Mostafa, D. A. Successful management of severe gummy smile using gingivectomy and botulinum toxin injection: A case report. Int J Surg Case Rep. Amsterdam, v. 42, p. 169-174, 2018.In: Trentin, MS et al. Correção de assimetriadento-gengival com finalidade estética. Relato de caso clínico. SALUSVITA. Bauru, v. 37, n. 3, p. 671-684, 2018

31-Amorim Lopes JC, Lopes RR, Santos SC. Cirurgia plástica periodontal empregada na correção do sorriso gengival. Relato de casos. J Bras OdontolClin 1999; 3(13):80-2.

32-Ramfjord SP, Ash MM. Gingivectomia. In: Periodontologia y periodoncia. 1ªed. Buenos Aires:Médica Panamericana; 1982. Cap.21. p.451-69. Sousa, C. P.;Garzon, A. C. D. M.; Sampaio, J. E. C. Estética periodontal: relato de um caso. Rev Bras Cir Period, v. 1, p. 262-267, 2003.bb. In: Álvaro NLA, Oliveira CMG. Gengivectomia e Gengivoplastia: Em Busca ao "Sorriso Perfeito". [acesso 14 set.2020] Disponível em: https://www.uninovafapi.edu.br/arquivos_academicos/repositorio_Biblioteca/odo ntologia/20191/ASPECTOS\%20GENGIVAI S\%20PARA\%20OTIMIZA \%C3\%87\%C3\%830\%20DO\%20SORRISO-\%20REVIS\%C3\%83O\%20DE\%2 0LITERATURA.pdf

33-Rossetti, E. P.; Sampaio, L. M.; Zuza, E. P. Correlação de assimetria dentogengival com finalidade estética: Relato de Caso. Revista Gaúcha de Odontologia. Campinas, v. 54, n. 4, p. 384-387, 2006. In: Trentin, MS et al. Correção de assimetria dento-gengival com finalidade estética. Relato de caso clínico. SALUSVITA. Bauru, v. 37, n. 3, p. 671-684, 2018.

34-Spada, CA et al. Cirurgia Plástica Periodontal para Recessão Múltiplas -Caso Clínico da Técnica dos Arcos de Saba-Chujfi. Ver Bras Cir Periodontia2003;1(4):298-303. [acesso 10 nov 2020] disponível: https://www.dtscience.com/wp-content/uploads/2015/10/Cirurgia- Pl\%C3\%A1sticaPeriodontal-para-Recess\%C3\%B5es-M\%C3\%BAltiplas-\%E2\%80\%93-Caso-Cl\%C3\%ADnico-da-T\%C3\%A9cnica-dos-Arcos-de-Saba-Chujfi.pdf

35-Carranza FA. A técnica da gengivectomia. In: Carranza FA, Newman MG. Periodontia clínica.8a ed. Rio de Janeiro: Rio de Janeiro; 1997. Cap.53. p.624-8. In: Sousa, C. P.; Garzon, A. C. D. M.; Sampaio, J. E. C. Estética periodontal: relato de um caso. Rev Bras Cir Period, v. 1, p. 262-267, 2003.bb. In: Álvaro NLA, Oliveira CMG. Gengivectomia e Gengivoplastia: Em Busca ao "Sorriso Perfeito".[acesso 14 set.2020] Disponível https://www.uninovafapi.edu.br/arquivos_academicos/repositorio_Biblioteca/odontologia/20191/ASPECTOS\%20GENGIVAIS\%20PARA\%20OTIMIZA $\%$ C3\%87 \% \%830\%20DO\%20SORRISO- \%20REVIS\%C3\%83O\%20DE\%20LITERATURA.pdf

36-Loi H, Nakata S, Counts AL. Influence of gingival display on smile aestheticsin Japanese. Eur J Orthod 2010; 32:633-637 In: Pinto, TB. Técnicas de Correção do Sorriso Gengival. [acesso 16 set.2020] Disponível em:https://repositorio.ul.pt/bitstream/10451/26239/1/ulfmd06056_tm_Tiffany_Pinto. pdf.

37-Morley J, Eubank J. Macroesthetic elements of smile design. J Am Dent Assoc 2001; 132(1):39-45. Sousa, C. P.; Garzon, A. C. D. M.; Sampaio, J. E. C. Estética periodontal: relato de um caso. Rev Bras Cir Period, v. 1, p. 262-267, 2003.bb. In: Álvaro NLA, Oliveira CMG. Gengivectomia e Gengivoplastia: Em Busca ao "Sorriso Perfeito". [acesso 14 set.2020] Disponível em: https://www.uninovafapi.edu.br/arquivos_academicos/repositorio_Biblioteca/odo ntologia/20191/ASPECTOS\%20GENGIVAIS\%20PARA\%20OTIMIZA\%C3\%87\%C3\%830\%20DO\%20SORRISO-

\%20REVIS\%C3\%83O\%20DE\%20LITERATURA.pdf. 